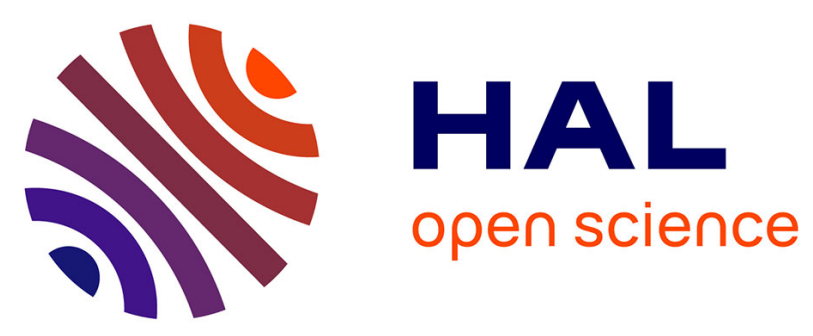

\title{
Compact infrared pinhole fisheye for wide field applications
}

\author{
Guillaume Druart, Nicolas Guérineau, Jean Taboury, Sylvain Rommeluère, \\ Riad Haïdar, Jérôme Primot, Manuel Fendler, Jean-Charles Cigna
}

\section{- To cite this version:}

Guillaume Druart, Nicolas Guérineau, Jean Taboury, Sylvain Rommeluère, Riad Haïdar, et al.. Compact infrared pinhole fisheye for wide field applications. Applied optics, 2009, 48 (6), pp.1104-1113. 10.1364/AO.48.001104 . hal-00700221

\section{HAL Id: hal-00700221 \\ https://hal-iogs.archives-ouvertes.fr/hal-00700221}

Submitted on 25 May 2012

HAL is a multi-disciplinary open access archive for the deposit and dissemination of scientific research documents, whether they are published or not. The documents may come from teaching and research institutions in France or abroad, or from public or private research centers.
L'archive ouverte pluridisciplinaire $\mathbf{H A L}$, est destinée au dépôt et à la diffusion de documents scientifiques de niveau recherche, publiés ou non, émanant des établissements d'enseignement et de recherche français ou étrangers, des laboratoires publics ou privés. 


\title{
Compact infrared pinhole fisheye for wide field applications
}

\author{
Guillaume Druart, ${ }^{1, *}$ Nicolas Guérineau, ${ }^{1}$ Jean Taboury, ${ }^{2}$ Sylvain Rommeluère, ${ }^{1}$ \\ Riad Haïdar, ${ }^{1}$ Jérôme Primot, ${ }^{1}$ Manuel Fendler, ${ }^{3}$ and Jean-Charles Cigna ${ }^{3}$ \\ 'ONERA, Chemin de la Hunière, 91761 Palaiseau Cedex, France \\ ${ }^{2}$ Institut d'Optique, Campus Polytechnique RD 128, 91127 Palaiseau Cedex, France \\ ${ }^{3}$ CEA-LETI/MINATEC, 17 Rue des Martyrs, 38054 Grenoble Cedex 9, France \\ ${ }^{*}$ Corresponding author: Guillaume.druart@onera.fr
}

Received 14 November 2008; revised 22 January 2009; accepted 27 January 2009; posted 29 January 2009 (Doc. ID 103930); published 13 February 2009

\begin{abstract}
The performances of a compact infrared optical system using advanced pinhole optics for wide field applications are given. This concept is adapted from the classical Tisse design in order to fit with infrared issues. Despite a low light gathering efficiency and a low resolution in comparison with classical lenses, pinhole imagery provides a long depth of field and a wide angular field of view. Moreover, by using a simple lens that compresses the field of view, the angular acceptance of this pinhole camera can be drastically widened to a value around $180^{\circ}$. This infrared compact system is named pinhole fisheye since it is based on the field lens of a classical fisheye system. (c) 2009 Optical Society of America

OCIS codes: $\quad 050.1970,110.0110,110.2970,110.3000,130.0130,130.3990$.
\end{abstract}

\section{Introduction}

A huge effort is made to produce miniature and cheap optical systems. The race for miniaturization leads to breakthroughs in optical design, such as multichannel optical systems inspired from the invertebrate vision [1-5], or simplified optical systems jointly optimized with an image processing to increase the image quality [6-8]. Moreover, the increase in sensitivity of modern detectors allows us to develop nonconventional optical components with interesting properties such as a long depth of field or tunable capabilities [9]. They thus allow the simplification and the miniaturization of traditional designs and can even replace all conventional optical elements in a system. For instance, a simple imaging system with a linear variable zoom can be made by only translating a diffractive axicon [10]. Such systems, without lenses, are called lensless imaging systems. The camera obscura or pinhole imagery is

0003-6935/09/061104-10\$15.00/0

(C) 2009 Optical Society of America the most trivial example of lensless imaging systems. Pinholes have been used for imaging purpose for centuries [11]. The Arabian scholar Ibn Al-Haithan in the 10th century used this device to safely observe solar eclipses, and in the 15th century, it was integrated by Della Porta in a dark room in one wall, called camera obscura. Despite its antiquity and apparent simplicity, pinhole imagery is still used in modern technical and scientific applications, essentially where the use of lenses is not possible or where angular resolution is not a major factor. They indeed offer several advantages over lens optics such as no absorption, complete freedom from linear distortion, a great depth of field, and a natural wide angular field of view (FOV) that can reach $70^{\circ}$. Characterizations and considerations for optimum performance have been widely discussed [12-17]. With the increase in sensitivity of modern detectors, applications using pinholes can be widened. Recently, Tisse has proposed a cheap, miniature, and wide FOV visual sensor that includes field-widened pinhole optics and most recent CMOS imager technology $[18,19]$. This optical system is dedicated to 
self-motion estimation, and it was designed in a topdown approach: the way of treating the optical information has led to the design of the optical system. We propose in this paper a transposition of this concept in the infrared spectral range. Indeed, the design has to be modified in order to fit with the constraints linked to the infrared spectral range. This optical system may be dedicated to security applications. In the first section, the optical performance of the pinhole in terms of angular resolution, FOV, and étendue are recalled. These criteria are useful to design a compact infrared pinhole fisheye in the second section. A way to widen the field of view of the pinhole in the infrared spectral range is described too. The performance of this optical system is then estimated by simulation and confronted by experiment in a third section.

\section{Description of the Performance of the Pinhole}

\section{A. On-Axis Considerations}

In this paper, the pinhole is a circular aperture of diameter equal to $s$ and illuminated by an incoherent wave. A point source placed at a distance $d$ from the pinhole is imaged by this latter at a distance $f$. These parameters are summarized in Fig. 1 . The corresponding point spread function (PSF) of this spherical wave imaged by the pinhole can be established by the modulus square of the amplitude of the field $U\left(\rho^{\prime}, f\right)$ diffracted by the pinhole at a distance $f$. Using the parabolic (Fresnel) approximation of the Rayleigh-Sommerfeld integral [20], $U\left(\rho^{\prime}, f\right)$ is given by the following equation:

$$
\begin{aligned}
U\left(\rho^{\prime}, f\right)= & U_{0} \int_{0}^{2 \pi} \int_{0}^{\frac{s}{2}} \operatorname{disc}\left(\frac{2 \rho}{s}\right) \exp \left(\frac{i \pi \rho^{2}}{\lambda}\left(\frac{1}{d}+\frac{1}{f}\right)\right) \\
& \times \exp \left(\frac{2 i \pi \rho . \rho^{\prime}}{\lambda f}\right) \rho \mathrm{d} \rho \mathrm{d} \varphi
\end{aligned}
$$

where

$$
U_{0}=\frac{i}{\lambda f} \exp (i k f) \exp \left(\frac{i \pi \rho^{2}}{\lambda f}\right)
$$

and $\operatorname{disc}(2 \rho / \mathrm{s})$ is the transmittance of the pinhole equal to 1 if $\rho \leq \mathrm{s} / 2$ and 0 elsewhere. The PSF according to $f$ of a plane wave, i.e., when $d \rightarrow \infty$, of

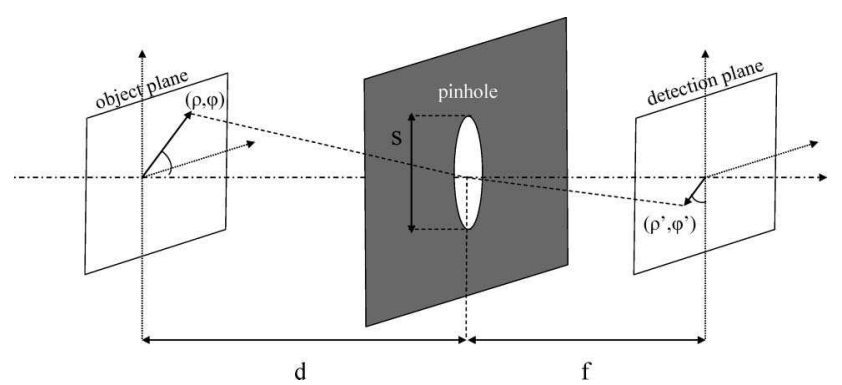

Fig. 1. Illustration of the parameters used for the analysis of the pinhole. wavelength $\lambda=4 \mu \mathrm{m}$ going through a pinhole of diameter $260 \mu \mathrm{m}$ has been simulated, and its transverse intensity profile is given in Fig. 2. Its Fourier transform yields the optical transfer function (OTF) whose modulus is called a modulation transfer function (MTF). Studies on the MTF give information on the quality of an optical system, such as its angular resolution, by assessing its cutoff frequency. The variations of the frequencies at $0 \%, 5 \%, 10 \%$, and $15 \%$ of the MTF versus $f$ at the configuration described above are also given in Fig. 2. We notice in this figure that the PSF of the pinhole can be smaller than the diameter of this latter for certain values of $f$, and there exists a position $f$ that maximizes the cutoff frequency of a pinhole camera. A thorough study on Eq. (1) can explain the variation of the cutoff frequency observed in Fig. 2. Indeed Eq. (1) shows that the PSF of the pinhole at a distance $f$ is the Fourier transform of a disc degraded by the presence of defocus (see [12]). This defocus is expressed by the aberrant phase $\phi_{\text {aberr }}$ :

$$
\phi_{\mathrm{aberr}}(\rho, \varphi)=\frac{i \pi \rho^{2}}{\lambda}\left(\frac{1}{d}+\frac{1}{f}\right)
$$

It is convenient, as we see below, to introduce the parameter $\beta$ given by the following expression:

$$
\beta=\frac{s^{2}}{\lambda}\left(\frac{1}{d}+\frac{1}{f}\right)
$$

Indeed this parameter is proportional to the maximal amount of aberration:

$$
\max \left(\phi_{\text {aberr }}\right)=\frac{\pi}{4} \beta
$$

The quality of the PSF of a pinhole can be generally evaluated by the parameter $\beta$ and therefore evaluated independently of the parameters $s, f, d$, and $\lambda$. The properties on the MTF at specific values of $\beta$ on the simulation described above can easily be generalized to other configurations. The PSF of the pinhole is an Airy function when $\phi_{\text {aberr }} \ll 1$. Its cutoff frequency $\nu_{c 1}$ is thus defined by the following relation:

$$
\nu_{c 1}=\frac{s}{\lambda f} .
$$

When the object is at infinity, i.e., when $d \rightarrow \infty$, the cutoff frequency $\nu_{c 1}$ can be written by Eq. ( $\left.\underline{4}\right)$ as follows:

$$
\nu_{c 1}=\frac{\beta}{s}
$$

The frontier between the Fraunhofer zone, when the PSF is an Airy function, and the Fresnel zone, when the PSF is degraded by defocus, is defined 


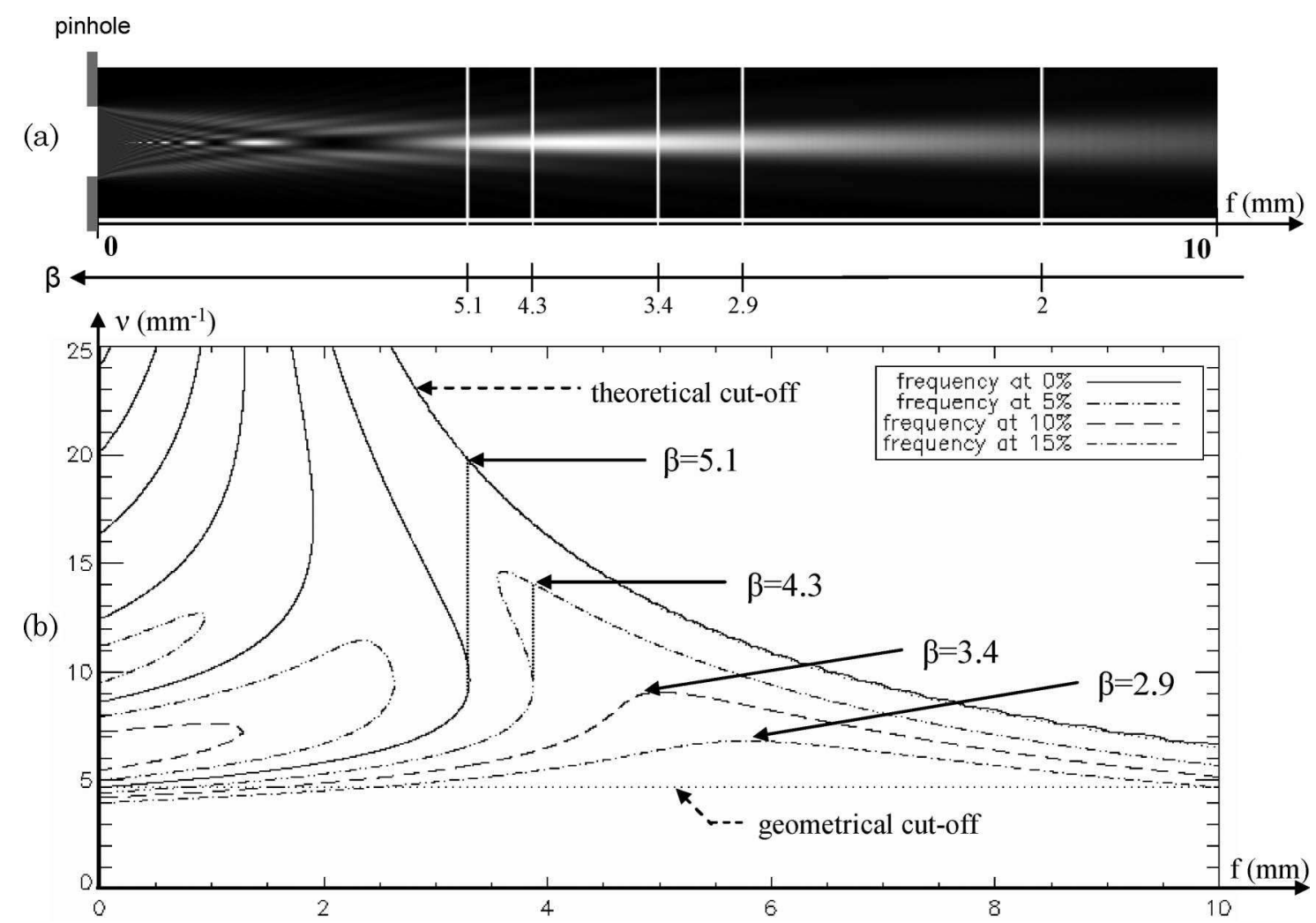

Fig. 2. (a) Illustration of the transverse intensity according to $f$ of a plane wave of wavelength $\lambda=4 \mu \mathrm{m}$ going through a pinhole of diameter $260 \mu \mathrm{m}$. (b) Variations of the frequencies at $0 \%, 5 \%, 10 \%$, and $15 \%$ of the normalized MTF versus distance of propagation $f$ for a pinhole of diameter $260 \mu \mathrm{m}$ at $\lambda=4 \mu \mathrm{m}$.

by $\max \left(\phi_{\text {aberr }}\right)=1$, i.e., by $\beta=1.27$. For $\beta>1.27$, the quality of the MTF, in comparison with the ideal MTF, becomes degraded up to the appearance of a cutoff frequency $\nu_{c 2}$ smaller than the theoretical cutoff $\nu_{c 1}$. This can be observed in Fig. 2. For $\beta>5.1$, the MTF possesses sidelobes, i.e., several frequencies at the corresponding value of 0 and smaller than the theoretical cutoff $\nu_{c 1}$. At these values of $\beta$, the quality of the image is drastically degraded because of the small cutoff frequency $\nu_{c 2}$ and the various inversions of contrast. An area for imagery application can thus be defined for the pinhole. It verifies the relation $\beta<5.1$, and the cutoff frequency in this area corresponds to $\nu_{c 1}$ given in Eqs. (6) and (7). This latter shows that, for a fixed value of $s, \nu_{c 1}$ increases with $\beta$. An optimal value $\beta_{\text {opt }}$ that, at the same time, maximizes $\nu_{c 1}$ and preserves a good quality of the MTF can, however, be established. The RMS criterion can be used to find this compromise. It is indeed a fine criterion to assess the quality of a PSF [21]. The root mean square $\Delta$ RMS is calculated in the following way:

$$
\Delta \mathrm{RMS}=<\phi_{\text {aberr }}^{2}>-<\phi_{\text {aberr }}>^{2},
$$

where

$$
\left\langle\phi_{\text {aberr }}\right\rangle=\frac{\int_{0}^{2 \pi} \int_{0}^{s / 2} \phi_{\text {aberr }}(\rho, \varphi) \rho \mathrm{d} \rho \mathrm{d} \varphi}{\int_{0}^{2 \pi} \int_{0}^{s / 2} \rho \mathrm{d} \rho \mathrm{d} \varphi} .
$$

In the case of the pinhole, $\Delta \mathrm{RMS}$ is equal to

$$
\Delta \mathrm{RMS}=\pi \beta \frac{\sqrt{3}}{24} .
$$

Applying the Marechal criterion [22], if the value of the $\Delta$ RMS is greater than $\pi / 7$, then the optical system is limited by defocus. The value of $\pi / 7$ corresponds to a Strehl ratio equal to $80 \%$. In this condition, we obtain $\beta=2$. This value is consistent with the quarter wave criterion too, as demonstrated by Mielenz in [15], and it is called the Petzval constant. He concludes, however, that there is a wide range of $\beta$ and, in particular, higher values of $\beta$ that give acceptable sharp images for practice purposes. The ambiguity of founding the right value $\beta$ reveals the long focal depth property of the pinhole. The value $\beta=2$ is a good criterion for classical imagery requiring a good MTF, but in the case of specific imagery, i.e., imagery of good contrast objects, we can choose a higher value of $\beta$ that leads to a more degraded MTF but with a higher cutoff frequency. Other criteria have been proposed to establish the optimal value of $\beta$. For instance, $\beta$ can be chosen in order to maximize the frequency defined from the value of the MTF at 5\% (see [12]) or at $10 \%$ (see [14]). Figure 2 shows that the optimal values of $\beta$ for the $5 \%$ criterion and the $10 \%$ criterion are, respectively, equal to 4.3 and 3.4 and are thus different. The $10 \%$ criterion gives a value of $\beta$ close to the one 
experimentally established by Rayleigh $(\beta=3.6$, [12]). The quality of the MTFs for three values of $\beta$ $(\overline{5.1}, 3.4$, and 2 ) is given in Fig. 3 and is compared with the ideal MTF. In the imagery area, the angular resolution IFOV can be established by Eq. (6). It is linked to the focal length of the optical system and the cutoff frequency $\nu_{c 1}$ of its MTF (generally expressed in $\mathrm{mm}^{-1}$ ) by the relation

$$
\mathrm{IFOV}=\arctan \left(\frac{1}{f \nu_{c 1}}\right)
$$

In the case of a pinhole in its imagery area, IFOV is thus approximately equal to

$$
\mathrm{IFOV}=\frac{\lambda}{s} .
$$

We notice that in the imagery area, IFOV does not depend on $f$ and is inversely proportional to $s$. Even if the pinhole is IFOV invariant along $f$, Eq. (4) shows that maximizing $\beta$ maximizes the compactness of the optical system. The IFOV invariance is another argument to explain the long focal depth of the pinhole. Equation (12) shows that the angular resolution, in the imagery area of a pinhole, depends on the wavelength $\lambda$ too. The difference in angular resolution between two wavelengths is given by the relation

$$
\frac{\Delta \mathrm{IFOV}}{\mathrm{IFOV}_{\mathrm{Avg}}}=\frac{\Delta \lambda}{\lambda_{\mathrm{Avg}}}
$$

where $\mathrm{IFOV}_{\mathrm{Avg}}$ and $\lambda_{\mathrm{Avg}}$ are, respectively, the mean values of IFOV and of $\lambda$ in relation with a given spectral range $\left[\lambda_{\min }, \lambda_{\max }\right]$. For an infrared spectral range equal to $[3,5 \mu \mathrm{m}]$ and in the case of a pinhole of diameter $260 \mu \mathrm{m}$, we obtain $\Delta \mathrm{IFOV}=0.44^{\circ}$ and $\mathrm{IFOV}_{\mathrm{Avg}}=0.88^{\circ}$. The difference of resolution is not negligible when viewing a scene in a large spectrum,

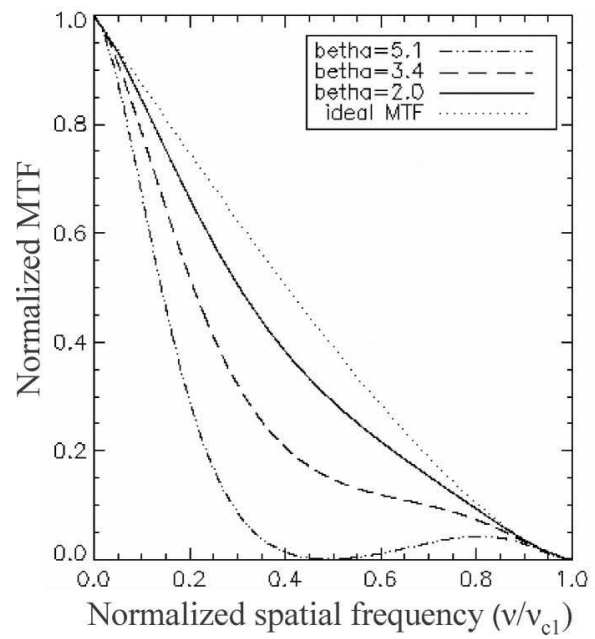

Fig. 3. Comparison of the MTFs of a pinhole of diameter $260 \mu \mathrm{m}$ at $\lambda=4 \mu \mathrm{m}$ at configuration $\beta=5.1,3.8$, and 2 with the ideal MTFs. and the polychromatic MTF will deeply depend on the spectrum of the source. Equation (4) shows that a conjugation formula between the object and the image can be established as follows:

$$
\frac{1}{d}+\frac{1}{f}=\beta \frac{\lambda}{s^{2}}
$$

This formula is precisely the one used in the theory of lenses [23], considering that the focal length of the pinhole is equal to $\beta \lambda / s^{2}$. Moreover, in the same way as an ordinary camera, the irradiance of the pinhole in its imagery area depends on the numerical aperture $N$ [16]:

$$
N=\frac{f}{s},
$$

and for an object at infinity $(d \rightarrow \infty)$, the étendue $G$ is proportional to $N$ as follows:

$$
G=\frac{\pi A_{d}}{4 N^{2}},
$$

where $A_{d}$ is the surface of the pixel and where $N$ can be written by Eq. (ㄴ) by the following equation:

$$
N=\frac{s}{\lambda \beta}
$$

We notice that, for given $s$ and $\lambda, G$ is maximized by maximizing $\beta$.

\section{B. Off-Axis Considerations}

Aberrationlike phenomena, similar to field curvature and astigmatism, have been shown in Refs. [12,16,23], the cause of which is the change of the apparent aperture and the increase of the path length when increasing the incidence angle $\theta$. Simple geometric considerations can be used to assess the influence of these aberrations on the angular resolution and on the étendue $G$. When the tangential incidence angle of the wave is equal to $\theta$, this latter sees the pinhole as an oval-shaped aperture defined by a radial diameter $s_{r}=s$ and a tangential diameter $s_{t}=s \times$ $\cos (\theta)$. As the detector is perpendicular to the optical axis of the pinhole, the path length of the off-axis ray is greater than the on-axis ray by a factor $1 / \cos \theta$ $\left(f^{\prime}=f / \cos \theta\right)$. Moreover, the intensity pattern falls obliquely onto the detector and therefore covers an area $1 / \cos \theta$ larger than the equivalent on the axis. These three effects tend to reduce the cutoff frequency of the off-axis MTF. Indeed Eq. (ㅁ) becomes

$$
\begin{aligned}
\nu_{t} & =\cos ^{3}(\theta) \frac{s}{\lambda f}, \\
\nu_{s} & =\cos (\theta) \frac{s}{\lambda f},
\end{aligned}
$$

and these equations reveal a decrease of the tangential IFOV: 


$$
\begin{gathered}
\mathrm{IFOV}_{t}=\cos ^{2}(\theta) \frac{\lambda}{s}, \\
\mathrm{IFOV}_{s}=\frac{\lambda}{s} .
\end{gathered}
$$

Moreover, these three effects combine to reduce also the photometric performance of the pinhole, and the étendue in oblique incidence is given by the well known $\cos ^{4} \theta$ law:

$$
G(\theta)=G \cos ^{4} \theta .
$$

We notice that the photometry is more degraded by the off-axis aberration than the angular resolution. Since the photometry is critical for pinhole imagery, the latter will limit the field of view (FOV) of the pinhole. The decrease of the étendue in oblique incidence in comparison with the on-axis étendue is illustrated in Fig. 4. If we limit the maximal off-axis étendue to half the value of the on-axis étendue, the accepted FOV of the pinhole is equal to $66^{\circ}$. The FOV of a pinhole could be drastically increased by using a curved detector [24]. Indeed, curved detectors could be a good way to attenuate the off-axis aberrations of the pinhole since the path length would be the same and all rays would fall perpendicularly onto the detector. This would result in an improvement of the étendue that would be degraded by a factor of only $\cos \theta$ (see Fig. 4). Using the same criterion as above, the pinhole could then accept a FOV of $120^{\circ}$. Finally, Selwyn demonstrated that the images produced by the pinhole are free from distortion [23].

\section{Proportion of a Pinhole Camera}

In Subsection 2.B, a criterium to choose the maximal accepted FOV for a pinhole has been established. To design a pinhole camera, a FOV less than or equal to $\mathrm{FOV}_{\text {max }}$ is fixed. Then the focal length of this pinhole camera can be established by the size det of the focal plane array and the chosen FOV by the following equation:

$$
f=\frac{\operatorname{det}}{2 \tan (\mathrm{FOV} / 2)}
$$

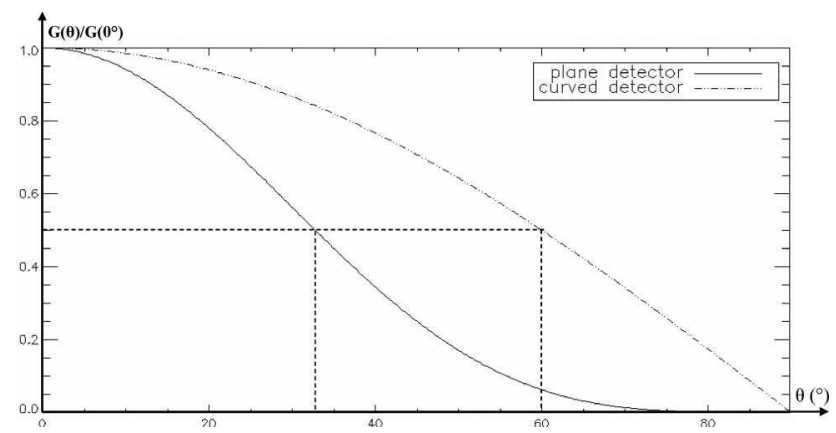

Fig. 4. Evolution of the étendue versus field angle $\theta$ in the case of a pinhole with a plane detector and with a curved detector.
When $f$ is determined, we have to choose the highest pinhole diameter $s$ in order to maximize IFOV and $G$. These are achieved by maximizing $\beta$ as suggested by Eqs. (7) and (17). However, a compromise has to be found on $\beta$, i.e., on $s$, in order to work in the pinhole imagery area and with a satisfactory MTF. As explained in Subsection 2.A, the optimal value $\beta_{\text {opt }}$ for classical imagery is equal to 2. Nevertheless, for specific applications with high contrasted objects, we accept working on more degraded MTF and, in this paper, we choose a $\beta_{\text {opt }}$ equal to

$$
\beta_{\mathrm{opt}}=3.4 \text {. }
$$

However, at $s$ and $f$ fixed, Eq. (4) indicates that condition (24) cannot be satisfied for each working wavelength $\lambda$ and for each wanted depth of field $d$. As we see in Subsection 3.C, the polychromatic PSF can be dramatically degraded if for some configurations of $\lambda$ and $d$ the pinhole works out of its imagery area. To avoid this case, the optimal diameter of the pinhole $s_{\text {opt }}$ must be designed in relation with the smallest wavelength of the spectral range of the system and with the highest wanted depth of field $d_{\text {min }}$ in order to be sure that the pinhole camera will work in its imagery area for all the other configurations. The optimal diameter $s_{\text {opt }}$ is thus given by the following relation:

$$
s_{\mathrm{opt}}^{2}=\frac{d_{\mathrm{min}}}{d_{\min }+f} \beta_{\mathrm{opt}} \lambda_{\min } f
$$

For all the other configurations, the corresponding $\beta$ will be smaller than $\beta_{\text {opt }}$ and will lead to a decrease of IFOV and $G$.

\section{Design of a Compact Infrared Pinhole Fisheye}

\section{A. How to Widen the FOV of the Pinhole Camera?}

We have seen in Section 2 that the maximal accepted FOV of a pinhole is around $66^{\circ}$. Some tricks have been proposed in the visible spectral range to extend the accepted FOV of a pinhole camera to $180^{\circ}$. The main principle is to use the refraction of a glass surface to reduce rays from a $180^{\circ} \mathrm{FOV}$ cone to a smaller FOV cone. The simplest way to do that is to use the refraction of a glass plate. Wood proposed to immerse the space between the pinhole and a photographic plate in a tank of water [25]. Franke's widefield camera [26] uses a hemispherical surface on the back of the pinhole to avoid immersing all the space between the pinhole and the detector [see Fig. 5(a)]. Indeed, the light cone is compressed on the first air-glass interface, and this compression is kept on the second glass-air interface because the light rays are everywhere perpendicular to the hemisphere and therefore undergo no refraction. Hsu proposed a different approach for widening the field of view of the pinhole [27]. He used a paraboloidal mirror and a pinhole placed at the focal point of this mirror. A photographic plate is placed in the focal plane. This 
method suppresses the problem of chromatic aberration due to refraction and the problem of intensity loss due to absorption. It diminishes the off-axis aberrations since the path length is uniform for all rays. However, this method is difficult to transpose to classical architectures of detection. Tisse in [18] proposed an improvement of the Franke's widefield camera by placing an additional plano-convex spherical lens cemented on the top of the pinhole. This camera is illustrated in Fig. 5(b). Tisse's camera results in less reflectance at the external air-glass interface and in a lower radial distortion. In the case of applications in the infrared spectral range $([3,5 \mu \mathrm{m}]$ for instance), this camera is, however, not adapted. Indeed, the lens and the half-ball have to be stuck together on the plate containing the pinhole, and the volume of air between the two optics at the level of the pinhole has to be filled with a glue with an index that matches the ones of these optics, in order to avoid total reflection on the glass-air interface. Unfortunately, glues in infrared are seldom or nonexistent. Usually infrared optics are not stuck together and are only placed side by side. A volume of air between these two lenses will degrade considerably the performance of the system, especially when the optics have a high index of refraction. For instance, the index of refraction of germanium is equal to 4 , and total reflections on the glass-air interface appear beyond an incidence angle of $14.5^{\circ}$, which is far below the limit of $45^{\circ}$ in Tisse's widefield camera. Moreover, as the photometry of pinholes is low, we will favor cooled detectors of HgCdTe technology, for example, instead of uncooled detectors like microbolometers, which are for the moment less sensitive. These cooled detectors have to be integrated in a cryostat, and their FOV is limited with a cooled diaphragm in order to diminish the background shot noise. In a problematic of miniaturization, the possibility of integrating the optical system directly in the cryostat is very interesting. We propose in this paper a modified version of Tisse's widefield camera in order to integrate this system in the cryostat and in order to fulfill all the constraints inherent to infrared system design. Instead of using a lens and a half-ball, we decide to dig in the first lens the volume of the

half-ball as described in Fig. 5. The lens becomes a divergent meniscus where the first surface of radius $R_{1}$ is used to compress the field and where the second surface of radius $R_{2}$ centered on the pinhole is used to avoid total refraction. Moreover, this new design can be integrated in the cryostat, where the meniscus becomes the window of the cryostat and where the pinhole becomes the cold diaphragm. The divergent meniscus is in fact similar to the first lens in a fisheye system. Indeed a classical fisheye system is composed of a first lens used to compress the field of view and a focusing part composed of an assembly of lenses and a diaphragm $[28,29]$. The number of lenses in the focusing part depends on the numerical aperture $N$ of the optical system. The higher $N$ is, the fewer lenses are needed for a diffraction-limited system. The advance-pinhole camera described above can thus be considered as an upper limit of a classical fisheye when $N$ is so high that no lenses are needed to have a diffraction-limited system. The diaphragm and "focusing function" are realized by a simple pinhole; that is why our compact infrared optical system is called a pinhole fisheye.

\section{B. Proportion of a Compact Pinhole Fisheye}

The different radii $R_{1}$ and $R_{2}$ of the field lens have to be established in order to obtain the wanted compressed field and to verify the wanted size of the optical system. We define $T$ as the distance between the top surface of the lens and the pinhole. The size of the optical system is thus defined by $T+f$. The field lens compresses the FOV of the optical system to a FOV equal to $\theta$ at the level of the pinhole. Using the notation of Fig. 6, we obtain the following relations that link $R_{1}$ to the optical size and the wanted compressed field:

$$
\begin{aligned}
& \sin \left(\theta_{a}-\frac{\mathrm{FOV}}{2}\right)=n \sin \left(\theta_{a}-\frac{\theta}{2}\right), \\
& \frac{T}{R_{1}}=\left[\cot \left(\frac{\theta}{2}\right)+\tan \left(\frac{\theta_{a}}{2}\right)\right] \sin \left(\theta_{a}\right) .
\end{aligned}
$$

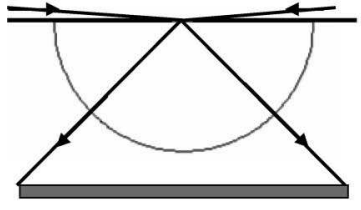

(a)

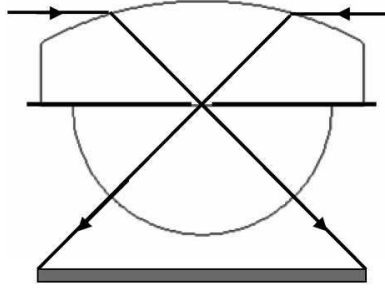

(b)

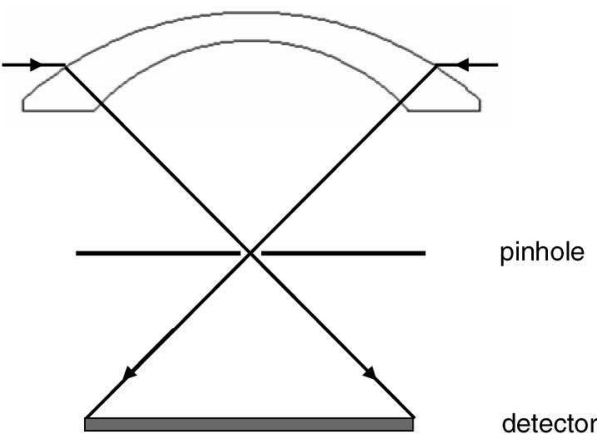

(c)

Fig. 5. Different designs to widen the field of view of a pinhole: (a) Franke's camera, (b) Tisse's camera, and (c) recommended configuration, called the pinhole fisheye. 


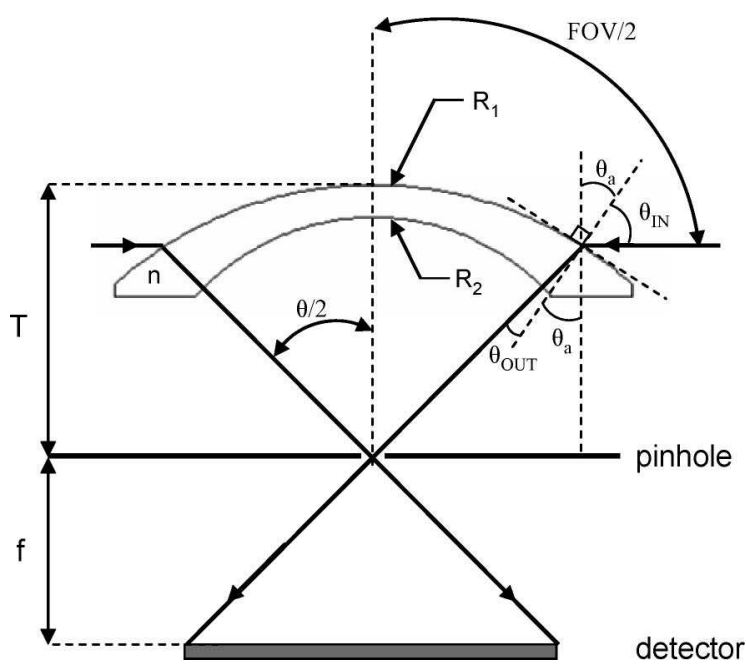

Fig. 6. Annotation of the pinhole fisheye.

The proportion of the field lens is similar to the one of the plano-convex lens describe by Tisse, and equations given in [18] to design this lens can thus be deduced from Eqs. (26) and (27). We, however, prefer the above equations since the evaluation of $R_{1}$ is more straightforward. There are less constraints on $R_{2}$ : indeed, $R_{2}$ must be inferior to $R_{1}$, and it is preferable that the smallest thickness of the lens $e=T-$ $R_{2}$ satisfies the relation $e>\phi / 10$ for optomechanical reasons, where $\phi$ is the diameter of the lens. The actual imaging device of the pinhole fisheye is the pinhole, the lens only increases the field of view and does not itself project an image. The performance of the pinhole fisheye, such as the cutoff frequency and the photometry, can thus be estimated by the relations given in Section 2. However, the divergent property of the fisheye lens has to be taken into account for the proportion of the pinhole. Indeed, the fisheye lens has an influence on the imagery area of the pinhole since the intermediate image of an object formed by the fisheye lens is at a finite distance $d$. In the case of an infinite point source, the intermediate image will be at a distance $d$ before the pinhole given by the following equation:

$$
d=\left|f_{2}\right|+T-e / 2,
$$

where $f_{2}$ is the focal length of the field lens given by the relation

$$
\frac{1}{f_{2}}=(n-1)\left(\frac{1}{R_{1}}-\frac{1}{R_{2}}\right)\left(1+\frac{n-1}{n} \frac{T-R_{2}}{R_{1}-R_{2}}\right),
$$

where $n$ is the index of refraction of the lens. We then define the factor of field compression as follows:

$$
C=\frac{\mathrm{FOV}}{\theta} .
$$

The expected angular resolution of the pinhole fisheye is thus given by Eq. (12) and Eq. (30) by the following equation:

$$
\mathrm{IFOV}_{\text {system }}=C \frac{\lambda}{s} .
$$

We have designed an omnidirectional compact infrared pinhole fisheye, having a field of view of $180^{\circ}$, a focal length of $4.8 \mathrm{~mm}$, and an optical size around $13 \mathrm{~mm}$. The field lens is in germanium $(n=4)$ and compresses the FOV of $180^{\circ}$ to a FOV of $67^{\circ}$. The factor of field compression of this system is thus equal to $C=2.7$. By Eqs. (26) and (27), we obtain $R_{1}=14.68 \mathrm{~mm}$. We choose $R_{2}=7.43 \mathrm{~mm}$ so that $e=1 \mathrm{~mm}$. Equation (29) gives the focal length on the optical axis of the field lens: $f_{2}=-4.54 \mathrm{~mm}$. Due to the influence of the field lens, the pinhole images an intermediate image placed at a distance $d=12.5 \mathrm{~mm}$ from the pinhole. The infrared pinhole fisheye works in the $[3,5 \mu \mathrm{m}]$ spectral range. The optimal diameter $s_{\text {opt }}$ of the pinhole can be established by Eq. (25) by taking the $\beta_{\text {opt }}$ of Eq. (24). We obtain the value $s_{\text {opt }}=188 \mu \mathrm{m}$. For the design of the pinhole fisheye, we have rather chosen an inexpensive offthe-shelf pinhole of diameter $200 \mu \mathrm{m}$, which is closed to $s_{\text {opt }}$ and that corresponds to a $\beta_{\text {opt }}=3.8$. The angular resolution of this pinhole fisheye is expected to be around $2.7^{\circ}$. In the case of an equivalent ideal optical system, having the same focal length and the same field compression as described above, the expected IFOV, limited by a pixel size pix and satisfying the Nyquist criterion, is given by the following relation:

$$
\mathrm{IFOV}_{\text {ideal }}=C \arctan \left(\frac{2 * \text { pix }}{f}\right) .
$$

In the configuration described above, the ideal IFOV for a classical infrared detector of pixel size pix $=30 \mu \mathrm{m}$ is equal to $1.9^{\circ}$, which is only 1.4 better than our compact pinhole fisheye. The expected numerical aperture $N$ of this pinhole fisheye is given by Eq. (17) and is equal to Eq. (24).

\section{Assessment of the Performance of the Optical System}

We have integrated the pinhole and the field lens into a cryostat. The mechanical assembly of the pinhole fisheye and the components that make up this latter are illustrated in Fig. 7. The pinhole replaces the cooled diaphragm, and the field lens replaces the window of the cryostat. The pinhole is cooled to $77 \mathrm{~K}$. We used an infrared focal plane array (IRFPA) of HgCdTe technology with a standard format, i.e., $320 \times 240$ pixels with a pitch of $30 \mu \mathrm{m}$. We acquired some images of the $180^{\circ}$ scene viewed by the pinhole fisheye (see Fig. 8). These images have been obtained with an integration time of $20 \mathrm{~ms}$ and after background subtraction. In Fig. 8(a), three people can be distinguished. In Fig. 8(b), a human face with glass close to the imager is represented. Both images illustrate the wide depth of focus as well as the wide field of view of the pinhole fisheye and its ability to 

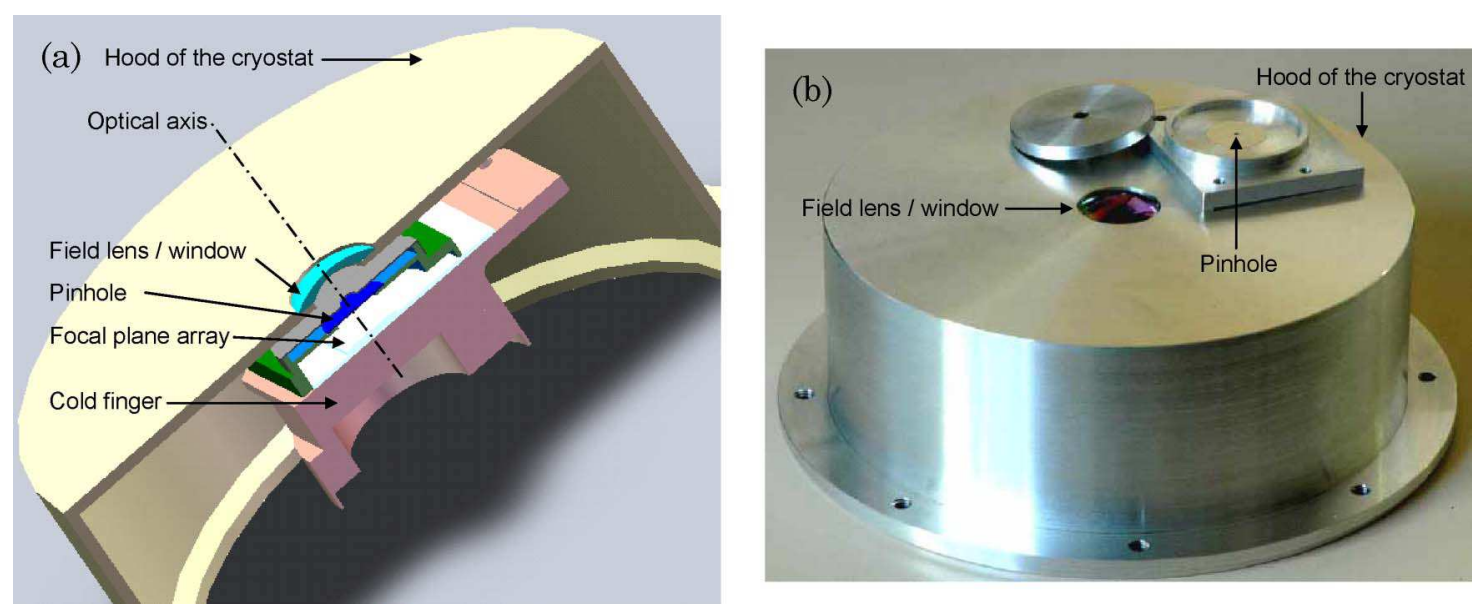

Fig. 7. (Color online) (a) Illustration of the mechanical assembly of the pinhole fisheye, (b) illustration of the different components of the pinhole fisheye.

spot people in a room. We have made a more accurate assessment of the quality of the pinhole fisheye by measuring experimentally the monochromatic PSFs of this camera and by comparing them with the simulated PSFs. In order to obtain the hyperspectral response of the pinhole fisheye, a commercial FTIR spectrometer (model Bruker Equinox IFS55) is used. The general principle is to measure the responses of the detector to a temporally varying signal called an interferogram (see [30]). A fast Fourier transform of these interferograms yields a cube of images corresponding to the responses of the system to a succession of monochromatic plane waves. This method is thus an indirect means of measuring monochromatic PSFs. This study is illustrated in Fig. 9. Figures 9(a) and 9(b) compare respectively the experimental and simulated radial profiles of the PSFs at $\lambda=3 \mu \mathrm{m}$ and at $\lambda=5 \mu \mathrm{m}$. In Fig. 9(c), the transverse PSFs according to $\lambda$ obtained by simulation and experimentally are given in the case of a pinhole fisheye with the characteristics given in the Subsection 3.B. In these simulations, the influence of the field lens has been taken into account. The simulations agree fairly well with the experimental results, thus validating the model described in Section 2. In Fig. 10, we compare the polychromatic radial $\mathrm{PSF}$, obtained experimentally, from a point source at $1200^{\circ} \mathrm{C}$ of two pinhole fisheyes having pinholes with different diameters corresponding respectively to a configuration that takes into account the divergent effect of the field lens (i.e., $s=200 \mu \mathrm{m}$ ) and to a configuration that neglects the influence of this latter $(s=260 \mu \mathrm{m})$. The half-width of the PSF, in the case of $s=200 \mu \mathrm{m}$, is equal to $100 \mu \mathrm{m}$ and corresponds to a value between the ones of the monochromatic PSFs at $\lambda=3 \mu \mathrm{m}$ and at $\lambda=5 \mu \mathrm{m}$. This value agrees with the assessment of the angular resolution given in Eq. (12). However, the half-width of the PSF, in the case of $s=260 \mu \mathrm{m}$, is equal to $240 \mu \mathrm{m}$ and is a value higher that the one assessed by Eq. (12). This can be explained by the fact that the divergent effect of the field lens tends to decrease the imagery area of the pinhole to higher values of $f$. If the pinhole is optimized in a configuration without the field lens, the addition of a field lens may place the pinhole camera out of its imagery area for some wavelengths. This will result in a quick increase of the half-width of the polychromatic PSF, as illustrated in Fig. 10,
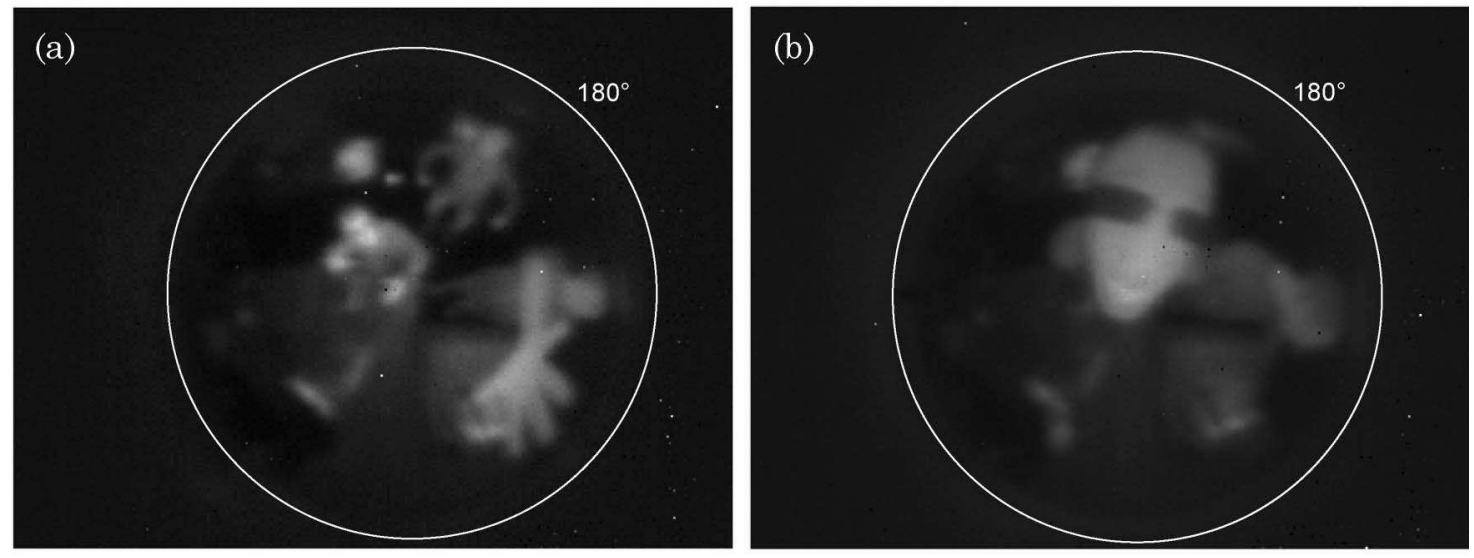

Fig. 8. Two images of the $180^{\circ}$ scene viewed by the pinhole fisheye. 

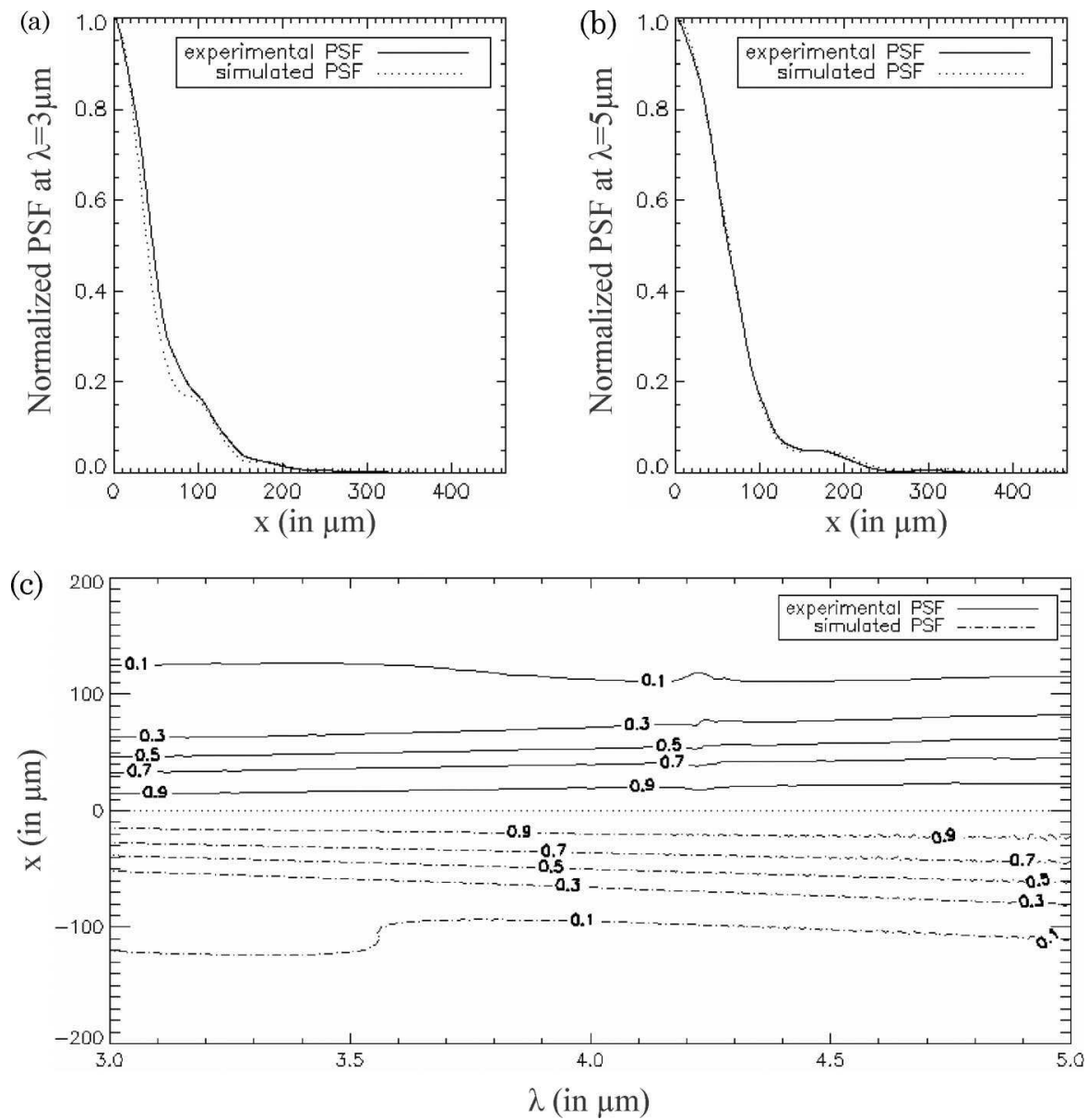

Fig. 9. Comparison between results obtained by simulation and experiment: (a), (b) the experimental and simulated radial profiles, respectively, of the PSFs at $\lambda=3 \mu \mathrm{m}$ and at $\lambda=5 \mu \mathrm{m}$. (c) Normalized transverse PSFs according to $\lambda$ for different values, obtained either experimentally (solid curves) or by simulation (dash dot curves). The pinhole fisheye has the characteristics described in Subsection 3.B.

and thus a quick decrease of the angular resolution. Therefore the divergent effect of the field lens must be taken into account in the proportion of the pinhole. Equation (25) shows that this effect, in comparison with the configuration without the field lens, tends to decrease the diameter of the pinhole for a given $f$ and a given $\lambda$ at $\beta_{\text {opt }}$ defined in Eq. (24). This results in a loss of angular resolution and sensitivity.

\section{Conclusion}

A compact infrared pinhole fisheye for surveillance applications has been designed. This optical system is composed of a pinhole as the only focusing element, and a field lens. This new design takes advantage of the classic mechanical environment of a cooled detector since the optical elements are totally integrated in the cryostat. The field lens replaces the window, and the pinhole replaces the cooled diaphragm. This design constitutes a breakthrough in the design of infrared optical systems using cooled detectors since optical elements are traditionally out of the cryostat. Therefore this new design maximizes the compactness of infrared systems using cooled detectors. The optical performances of a pinhole in terms of IFOV, FOV, and sensitivity have been established.
An imagery area of the pinhole has been demonstrated and a criterion has been established that maximizes compactness and sensitivity of a pinhole camera at a given focal length. The influence of the field lens on the imagery area has been determined too. The divergent effect of the field lens tends to decrease the diameter of the pinhole and thus to

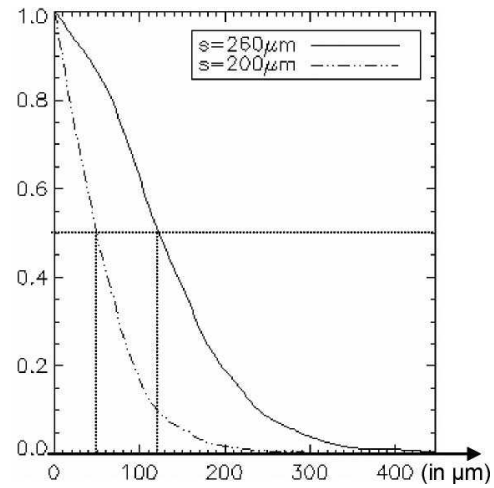

Fig. 10. Comparison between the experimental radial polychromatic PSFs of two pinhole fisheyes having a pinhole diameter of $200 \mu \mathrm{m}$ or $260 \mu \mathrm{m}$ and viewing a point source at $1200^{\circ} \mathrm{C}$. The field lens of these pinhole fisheyes is the one described in Subsection 3.B. The focal length of these cameras is equal to $4.8 \mathrm{~mm}$. 
decrease the angular resolution and the sensitivity of the camera. This infrared pinhole fisheye has been characterized experimentally, and similarities to the simulations have been shown. The pinhole fisheye benefits from the recent improvements of the sensitivity of cooled detectors, which can support optical systems with a high numerical aperture. Unfortunately, this design is not adapted for room temperature infrared arrays such as microbolometers since they require an optical system with a small numerical aperture, usually less than 2. Of course, the field lens can be taken away in order to benefit from the clandestine advantage of viewing through a pinhole, but this will be at the price of a smaller FOV (around $70^{\circ}$ ).

This work has been sponsored by the Délégation Générale de l'Armement (DGA) of the French Ministry of Defense.

\section{References}

1. J. Duparré, P. Dannberg, P. Shreiber, A. Bräuer, and A. Tünnermann, "Artificial apposition compound eye fabricated by micro-optics technology," Appl. Opt. 43, 4303-4310 (2004).

2. J. Duparré, P. Dannberg, P. Schreiber, A. Bräuer, and A. Tünnermann, "Thin compound-eye camera," Appl. Opt. 44, 2949-2956 (2005).

3. J. Duparré, P. Schreiber, A. Matthes, E. Pshenay-Severin, A. Bräuer, and A. Tünnermann, "Microoptical telescope compound eye," Opt. Express 13, 889-903 (2005).

4. V. Gubsky, M. Gertsenshteyn, and T. Jannson, "Lobster-eye infrared focusing optics," Proc. SPIE 6295, 62950F (2006).

5. G. Druart, N. Guérineau, R. Haïdar, E. Lambert, M. Tauvy, S. Thétas, S. Rommeluère, J. Primot, and J. Deschamps, "MULTICAM: a miniature cryogenic camera for infrared detection," in Micro-Optics, Proc. SPIE 6992, 699215 (2008).

6. J. Tanida, T. Kumagai, K. Yamada, S. Miyatake, K. Ishida, T. Morimoto, N. Kondou, D. Miyazaki, and Y. Ichioka, "Thin observation module by bound optics (TOMBO) concept and experimental verification,” Appl. Opt. 40, 1806-1813 (2001).

7. K. Kubala, E. Dowski, and W. T. Cathey, "Reducing complexity in computational imaging systems," Opt. Express 11, 21022108 (2003).

8. E. J. Tremblay, R. A. Stack, R. L. Morrison, and J. E. Ford, "Ultrathin cameras using annular folded optics," Appl. Opt. 46, 463-471 (2007).

9. Ch. Friese, A. Werber, F. Krogmann, R. Shaik, W. Monch, and H. Zappe, "New technologies for tunable micro-optics," Proc. SPIE 6993, 699306 (2008).
10. G. Druart, J. Taboury, N. Guérineau, R. Haïdar, H. Sauer, A. Kattnig, and J. Primot, "Demonstration of image-zooming capability for diffractive axicons," Opt. Lett. 33, 366-368 (2008).

11. Z. Jaroszewicz, A. Burvall, and A. T. Friberg, "AXICON-the most important optical element," Opt. Photon. News 16(4), 34-39 (2005).

12. K. Sayanagui, "Pinhole imagery," J. Opt. Soc. Am. 57, 10911099 (1967)

13. R. E. Swing and D. P. Rooney, "General transfer function for the pinhole camera," J. Opt. Soc. Am. 58, 629-635 (1968).

14. H. B. Edwards and W. P. Chu, "Graphic design of pinhole cameras," Appl. Opt. 18, 262-263 (1979).

15. K. D. Mielenz, "On the diffraction limit for lensless imaging," J. Res. Natl. Inst. Stand. Technol. 104, 479-485(1999).

16. M. Young, "Pinhole optics," Appl. Opt. 10, 2763-2767 (1971).

17. P. A. Newmann and V. E. Rible, "Pinhole array camera for integrated circuits," Appl. Opt. 5, 1225-1228 (1966).

18. C.-L. Tisse, "Low-cost miniature wide-angle imaging for self-motion estimation," Opt. Express 13, 6061-6072 (2005).

19. C.-L. Tisse and H. Durrant-Whyte, "Hemispherical eye sensor in micro aerial vehicles using advanced pinhole imaging system," in Proceeding of IEEE Conference on Intelligent Robots and Systems (IEEE, 2005), pp. 634-640.

20. J. W. Goodman, Introduction to Fourier Optics (McGraw-Hill, 1968), p. 30.

21. G. Druart, N. Guérineau, R. Haïdar, J. Primot, Pierre Chavel, and J. Taboury, "Nonparaxial analysis of continuous selfimaging gratings in oblique illumination,” J. Opt. Soc. Am. A 24, 3379-3387 (2007)

22. M. Born, E. Wolf, Principles of Optics, 6th ed. (Pergamon, 1989), Chap. 5, p. 203.

23. E. W. H. Selwyn, "The pin-hole camera," Photograph. J. B 90, 47-52 (1949).

24. S.-B. Rim, P. B. Catrysse, R. Dinyari, K. Huang, and P. Peumans, "The optical advantages of curved focal plane arrays," Opt. Express 16, 4965-4971 (2008).

25. R. W. Wood, Physical Optics (Dover, 1967), pp. 66-69.

26. J. M. Franke, "Field-widened pinhole camera," Appl. Opt. 18, 2913-2914 (1979).

27. T. Hsu, "Reflective wide-angle pinhole camera," Appl. Opt. 21, 2303-2304 (1982).

28. J. J. Kumler and M. L. Bauer, "Fish-eye lens designs and their relative performance," Proc. SPIE 4093, 360 (2000).

29. H. M. Spencer, J. M. Rodgers, and J. M. Hoffman, "Optical design of a panoramic, wide spectral band, infrared fisheye lens," Proc. SPIE 6342, 63421P (2007).

30. S. Rommeluère, R. Haïdar, N. Guérineau, J. Deschamps, E. de Borniol, A. Million, J.-P. Chamonal, and G. Destefanis, "Single-scan extraction of two-dimensional parameters of infrared focal plane arrays utilizing a Fourier-transform spectrometer," Appl. Opt. 46, 1379-1384 (2007). 